\title{
Q \\ Active Infrared Thermography for Non-Destructive Control for Detection of Defects in Asphalt Pavements
}

\author{
by M. Marchetti*, S. Ludwig*, J. Dumoulin**, L. Ibos*** ${ }^{* *}$ A. Mazioud ${ }^{* * *}$
}

*LRPC de Nancy, CETE de l'Esț, 71 rue de la Grande Haie, 54510 TOMBLAINẸ Nancy, France

**LPC, DM!, Route de Bouaye.- BP 4129, 44341 Bouguenais Cedex, France

${ }^{* * *}$ CERTES, Université Paris 12, 61 avenue du Générạl de Gaulle, 94010 Créteil Cedex, France

\begin{abstract}
Active infrared thermography has been used for the detection of sub surface defects in road materials. The step heating method was selected, and two different infrared cameras selected. Defects were detected with an heating phase as short as 60 seconds. Some images treatments were employed, and have been able to extract signal in spite of the porosity and of the high heterogeneity of a road material. Numerical model calculations were run, and have lead to experimental validations.
\end{abstract}

\section{Introduction}

Within the policy frame of the diagnosis and the maintenance of the French roads and bridges network, some investigations have been undertaken on non-destructive methods for roads pavements. Such methods could be applied on the field on real sites as much as possible. They should be able to detect hidden defects from the surface. Indeed, defects such as un-sticking zones between the top layer and the structural ones could induce a quick deterioration of the pavement surface (potholes ...). When the defect is punctual, or is located on a small road stretch, some efficient repair might solve the problem. Sometimes, it involved a larger number of kilometres. The renewal of the road is then the solution. Their detection as soon as possible has therefore a great importance and is an important challenge. It might implies some savings and avoid major traffic disruptions.

Active infrared thermography for the detection of defects has been used now for many years for non-destructive control of materials such as metals, composites and so on, as described in the literature [1]. The objective of this study was to evaluate to which extent this technique could be applied to road pavements. There is a large variety of materials used for road pavements, from asphalt to concrete. They might have different granular constitution and therefore different thermal and radiative properties. Their structures could be non to highly porous, and could include several layers. These variations could also appear within the same pavement due to construction constraints. These heterogeneities would induce some difficulties and challenges in the use of active thermography. Furthermore, one main obstacle would be to detect defects at great depths within a reasonable period of time. Such challenge would have to cope with the fact that the thermal properties gradients between the considered sound materials and the ones with defects could be weak.

The step heating method was selected, and two different infrared cameras selected. Defects were detected with an heating phase as short as 60 seconds. Some images treatments were employed, and have been able to extract signal in spite of the porosity and of the high heterogeneity of a road material. Numerical model calculations were run, and have lead to experimental validations.

\section{Description of the experimental setup}

\subsection{Asphalt pavement samples}

This preliminary study has consisted in setting a first measurement protocol for the use of active infrared thermography to detect defects within a laboratory frame. As a start, some semi-granular asphalt pavement material was considered. It is one of the most commonly used material either on national roads and highways. Samples were made of granular materials with a bitumen matrix. They consisted in parallelepipeds $(10 \mathrm{~cm} \times 18$ $\mathrm{cm} \times 50 \mathrm{~cm}$ ). Defects made of wood were included while manufacturing the road samples. During the thermal analysis, these defects were either were either left or removed. Two kinds of inclusions were therefore evaluated, which thermal conductivity and diffusivity (wood or air) were lower than the ones of the road material. The defects shapes were a parallelepiped and a pyramid. They were located at different depths under the samples surfaces $(1.3 \mathrm{~cm}$ and down to $6 \mathrm{~cm}$ ). These inclusions are inserted into the road pavement samples in such a way they should not thermally affect each other (see figure 1).

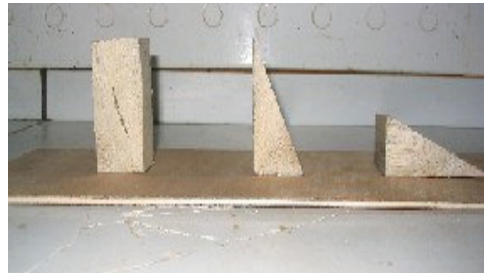

(a) shape and position of the three defects

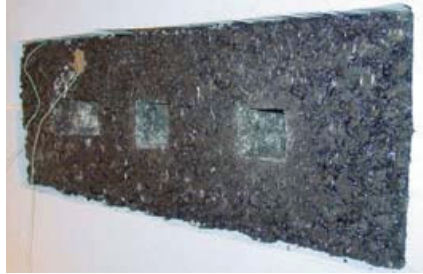

(b) Pavement sample rear side

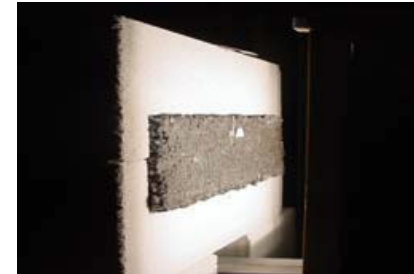

(c) Pavement sample front side

Fig. 1. Pictures of the defects included in the road pavement samples 


\subsection{Main characteristics of infrared cameras used}

Two different infrared cameras were employed to monitor the process of the surface temperature evolution during experiments. The first one was a CEDIP ${ }^{\circledR}$ camera JADE III (MWIR), and the second one was a FLIR ${ }^{\circledR}$ S65 (LWIR). In both cases, infrared images of the samples surface were taken at a given frequency. The characteristics of the cameras are summarized in tables 1 and 2.

Table 1. Main characteristics of the CEDIP. JADE I!I MWIR camera used

\begin{tabular}{|c|c|}
\hline Detector type & $\begin{array}{c}\text { InSb cooled by Stirling cycle } \\
\text { IRFPA of 320x240 sensitive elements }\end{array}$ \\
\hline Spectral bandwidth & $3.6-5.1 \mu \mathrm{m}$ \\
\hline Optical focal & $50 \mathrm{~mm}$ \\
\hline FOV & $11^{\circ} \times 8^{\circ}$ \\
\hline NETD at $\mathbf{3 0}{ }^{\circ} \mathbf{C}$ & $20-30 \mathrm{mK}$ \\
\hline Digitalization & 14 bits \\
\hline Pitch & $30 \mu \mathrm{m}$ \\
\hline Frame rate & Up to $46 \mathrm{~Hz}$ in full format \\
\hline
\end{tabular}

Table 2. Main characteristics of the FLIR ${ }^{\circledR}$ S6 5 LWIR camera used

\begin{tabular}{|c|c|}
\hline Detector type & $\begin{array}{c}\text { Microbolometer detector uncooled } \\
\text { IRFPA of 320x240 sensitive elements }\end{array}$ \\
\hline Spectral bandwidth & $7,5-13 \mu \mathrm{m}$ \\
\hline Thermal range used & $-40-+120^{\circ} \mathrm{C}$ \\
\hline Sensitivity & $0.08^{\circ} \mathrm{C}$ \\
\hline Accuracy & $\pm 2 \%$ of the measure \\
\hline FOV & $24^{\circ} \times 18^{\circ}(\mathrm{HxV})$ \\
\hline IFOV & $1,3 \mathrm{mrad}$ \\
\hline lower image acquisition frequency & $1 \mathrm{~Hz}$ \\
\hline Video output & Up to $50 \mathrm{~Hz} \mathrm{non} \mathrm{interlaced}$ \\
\hline
\end{tabular}

\subsection{Step heating bench - Test protocol}

The experimental setup consisted in two $500 \mathrm{~W}$ halogen lamps each, extensively described by Maldague [1] and in previous paper [2] and [3]. A reflector was used to get roughly constant flux density over the whole sample surface. The selected active thermography method was the step heating. It consisted in heating the sample for a given time length and then in proceeding to observations during the whole relaxation process. The heating phase lengths ranged between $60 \mathrm{~s}$ and up to 1 hour. The infrared camera is located at a distance of the sample surface consistent with its optical characteristics. The experimental setup is illustrated in figure2.

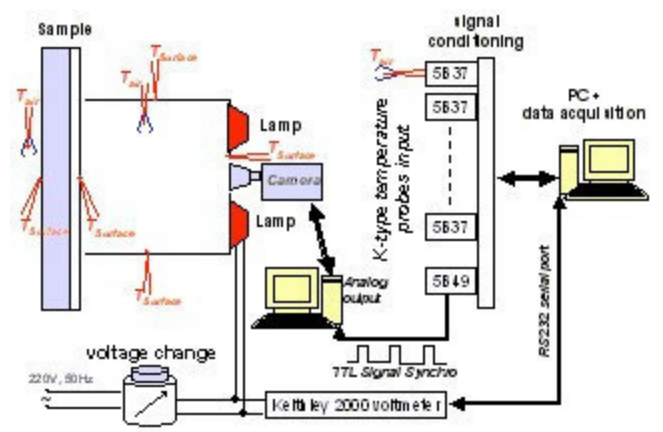

(a) full schematic description of the setup

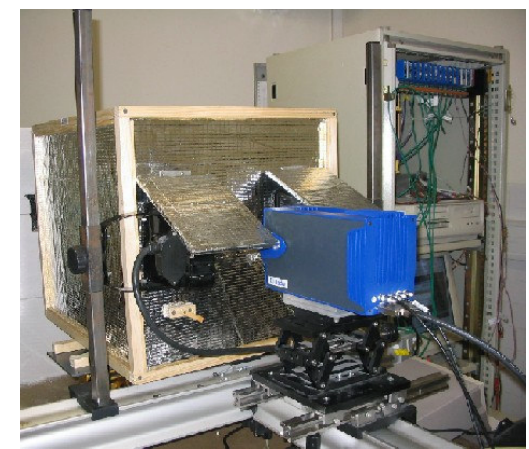

(b) picture of the experimental bench

Fig. 2. Step-heating experimentạl setup

\subsection{Numerical analysis of images and temperatures}

Temperatures on several different locations of the surface were monitored during the test. The monitoring is either conducted with thermocouples and with the infrared cameras.

The thermocouples were located on both sides of the samples: the one submitted to the heat flow, and the back one. There were used as a control of the procedure and for further numerical heat transfer calculations. Temperatures over the whole surface were monitored using infrared images. They gave an insight of the heating homogeneity and thermal response of semi-granular asphalt pavement material. at different stages of the tests. All data were collected and recorded using a LabVIEW ${ }^{\circledR}$ interface. 


\section{Active infrared control results}

Image analyses were used to locate the defects within the samples. They consisted in calculations of thermal contrast, in filtering the signal, and in generating contrast images. Defects located under the surface were easily found even with a short heating phase. Deeper the defect are, harder it is to detect them. The thermal behaviour of the structure was greatly affected by the defect presence around it. The nature of the samples (porosity, heterogeneity) did not seem to affect the ability of the technique to non destructive control of road structures.

\subsection{Infrared images of pavement course wearing samples}

Infrared images were taken at a 1 image /s rate in the worst case. Two main heat flux densities were applied to pavement sample during thermal pulse excitation $\left(770\right.$ and $\left.2620 \mathrm{~W} \cdot \mathrm{m}^{-2}\right)$ and one trial was made with a lower heat flux density of $220 \mathrm{~W} \cdot \mathrm{m}^{-2}$. Higher power density was used for lower pulse duration (1, 2, 3 or 5 min).during experimentation. Lower heat density was used for longer time experiment with two pulse durations of 30 min or 1 h. Infrared images were acquired during half an hour up to two hours.

Tests have shown that defects close to the surface could be detected during the heating phase using the infrared images. It was the case for the square one with a flat surface parallel to the heat flow For pyramid ones, no direct detection was observed, including for the one $1.3 \mathrm{~cm}$ below the sample surface. The heterogeneity of the pavement samples (materials and porosity) did not significantly affect the heat transfer within the structure. Some heating heterogeneity was recorded on samples edges. This is illustrated in figure 3 (a) with images acquired with FLIR $^{\circledR}$ S65 camera. On the other hand, images acquired with the CEDIP ${ }^{\circledR}$ Jade III allow to distinguish aggregates thermal behaviour from binder due to the spatial resolution used (see figure 3 (b)).

(a) FLIR ${ }^{\circledR}$ S65 images - heating duration 1 hour at $220 \mathrm{~W} . \mathrm{m}^{-2}$ - Two defects samples in wood

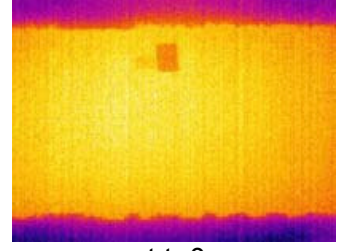

at $\mathrm{t}=0 \mathrm{~s}$

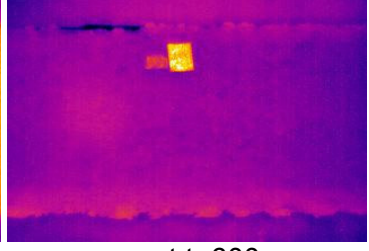

at $\mathrm{t}=600 \mathrm{~s}$

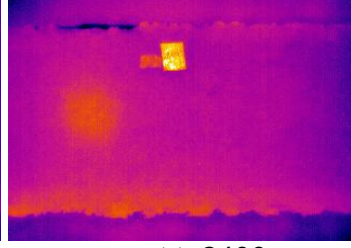

at $\mathrm{t}=2400 \mathrm{~s}$

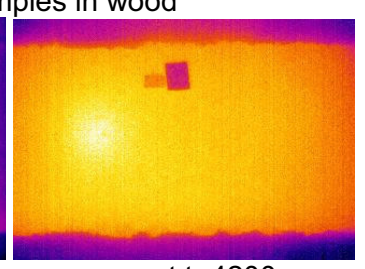

at $\mathrm{t}=4200 \mathrm{~s}$

(b) CEDIP ${ }^{\circledR}$ Jade III images - heating duration 1 minute at 2620 W. $\mathrm{m}^{-2}$ - Square defect in wood

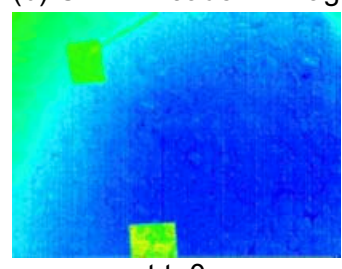

at $\mathrm{t}=0 \mathrm{~s}$

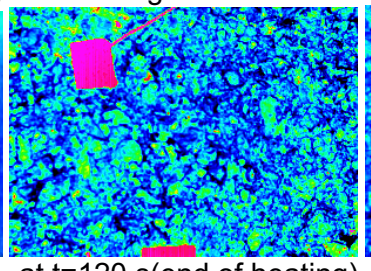

at $\mathrm{t}=120 \mathrm{~s}$ (end of heating)

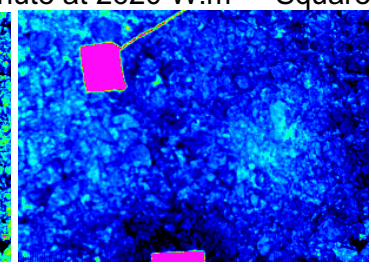

at $\mathrm{t}=200 \mathrm{~s}$

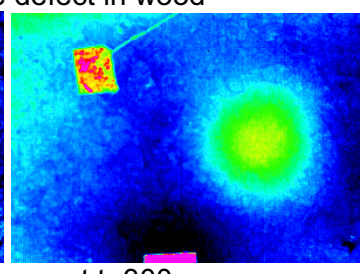

at $\mathrm{t}=300 \mathrm{~s}$

Fig. 3. Infrared images acquired with the different cameraș

\subsection{Thermal contrast calculations}

A defect can be detected if a thermal contrast is generated on the surface above it. Using the temperatures map of the infrared images acquired with the $\mathrm{FLIR}^{\circledR} \mathrm{S} 65$, a simple contrast calculation was undertaken. It consisted in establishing a ratio between temperature differences at a given moment with respect to what it was before the heating phase started. The temperature differences are taken above the defect area and the whole road sample (Eq. (1)). Such a choice is only valid if the defect area is small enough compared to one of a sound area.

$\operatorname{contrast}($ in $\%)=100 \cdot \frac{T_{\text {defect }}(t)-T_{\text {defect }}(t=0)}{T_{\text {image }}(t)-T_{\text {image }}(t=0)}$

For the images acquired with the CEDIP ${ }^{\circledR}$ Jade III contrast maps were computed by subtracting the Digital Level (DL) data collected during heat pulse excitation and thermal relaxation phase with the average Digital Level map acquired before heat pulse solicitation. In such approach no a priori on the location of the defect was used. Furthermore, in order to facilitate contrast map visual analysis or by image processing, an enhancement can be obtained by dividing these maps by the average value of the Digital Level map acquired before heat activation of the pavement sample.

An example of result obtained with data acquired with FLIR ${ }^{\circledR} S 65$ is given in figure 4 (a). As expected and as observed with many other materials, including the ones in civil engineering [4], a proper thermal contrast could be generated above a hidden defect when the proper test conditions are selected, and in spite of the highly heterogeneous structure of semi-granular asphalt pavement material. The best contrast was obtained $540 \mathrm{~s}$ after the heating phase started, for a $300 \mathrm{~s}$ long heating phase. In figure 4 (b) an example of results obtained with data acquired with CEDIP ${ }^{\circledR}$ Jade III is given. The first maximum peak of contrast at $60 \mathrm{~s}$ (end of pulse heating) can be 
explained by the difference of thermal behaviour of aggregates under a thin layer of binder and binder between aggregates. Contrast images presented in paragraph 3 in figure 7, obtained for $60 \mathrm{~s}$ of heat pulse with a density of 2620 W. $\mathrm{m}^{-2}$ over the square defect filled with air, illustrate this proposal of analysis. The second maximum peak of contrast is located round $250 \mathrm{~s}$ match is well correlated with the defect location and it is in a good agreement with contrast images presented in figure 7 which deals with the same trial.

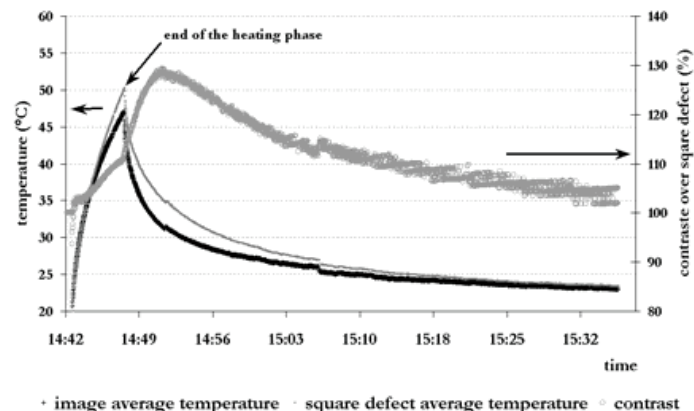

(a) data acquired with $\mathrm{FLIR}^{\circledR} \mathrm{S} 65$

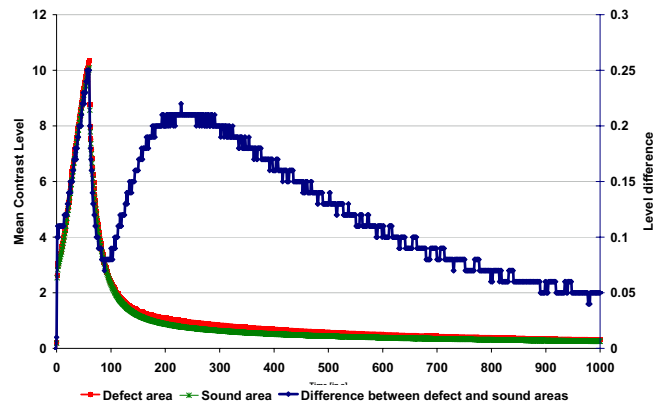

(b) data acquired with CEDIP ${ }^{\circledR}$ Jade III

Fig. 4. Example of computed thermạl contrașt evolution

\subsection{Numerical analysis of the infrared images}

\subsubsection{Contrast images}

As the contrast was calculated for the whole image, the same analysis could be run for each pixel of the numerical infrared images. The contrast images were generated using the method described by Maldague in [1]. Since the images obtained with the $\mathrm{FLIR}^{\circledR} \mathrm{S} 65$ camera presented an important noise, an average image was generated every 30 images. Then an image contrast was calculated. In such calculation, the temperature above the defect is compared to the one above a sound area. In the current situation, due to the heterogeneity of the pavement road samples (porosity and different materials), the definition of a sound area could be quite wide. Therefore, and as indicated before, the choice has been made to consider the whole surface sample. In such a description, the defect size is supposed small enough with respect of the surface. Some results of the contrast image calculations are given in figure 5 , in the case of a sample with two defects.

contrast: images $n^{\circ} 120$ to 149
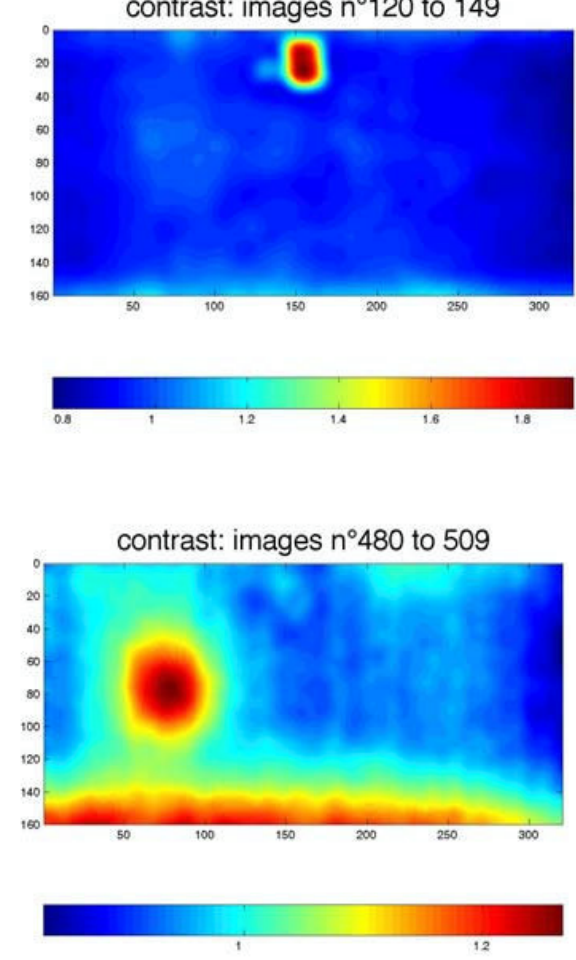

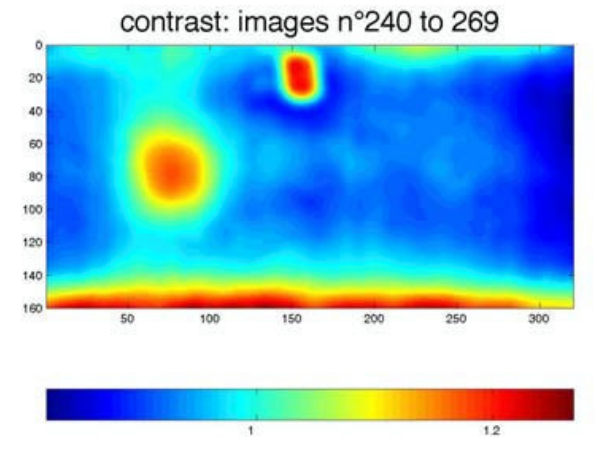

contrast: images $n^{\circ} 1020$ to 1049

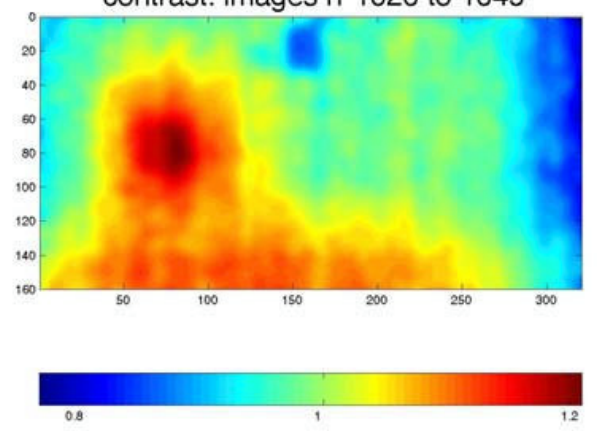

Fig. 5. FLIR. ${ }^{\circledR}$ S6 5 contrașt images (Average).- heating duration 5 mị ạt 2620 W. m. $^{-2}$.- Two defects pavemeṇt sample filled with pine wood 
As illustrated on Figure 5, the contrast remained high above the defect located $1.3 \mathrm{~cm}$ below the surface. Though this one already appeared during the heating phase in some conditions, its square shape is more easily recognizable on contrast images. On the other hand, the pyramid shaped defect, which top was either located 1.3 or $6 \mathrm{~cm}$ below the surface depending on the road sample, could not be detected on contrast images.

A line was chosen to intercept both defects. The thermal contrast over this line was calculated over time, and is illustrated on Figure 6.

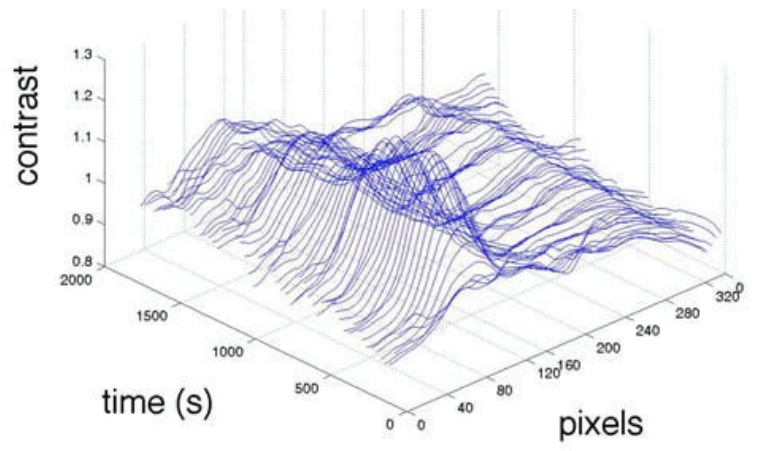

Fig. 6. Thermạl contrașt over a line vṣ. time

As shown on this figure, the square shape defect $1.3 \mathrm{~cm}$ below the surface could be easily identified, both in location and size (around pixel 80). The second one, pyramid-shaped, barely appeared. Some increase in the contrast appeared around pixel 240. But this could as well be attributed to some noise. The shape of the defect was such that the thermal wave could go around it without accumulation, as it was the case with the square one.

In figure 7, contrast map computed with images acquired with the CEDIP ${ }^{\circledR}$ Jade III for a pulse duration of 1 min with an heat density of 2620 W. $\mathrm{m}^{-2}$. Due to the optical configuration used only one defect signature can be followed during the experiment. As previously noticed in paragraph 2 at the end of the heating pulse aggregates contrast signature in the defect area or in sound area is quite of the same order of level. Shapes that can be extracted from this first contrast images match aggregates location under the thin film of bitumen. The signature of the square defect filled with air begins to have a detectable shape on contrast images less than two minutes after the ending of the heat pulse. The defect signature shape has a good homogeneity on contrast images and matches the square defect form at least 3 minutes after the ending of the heat pulse till 8 minutes after.

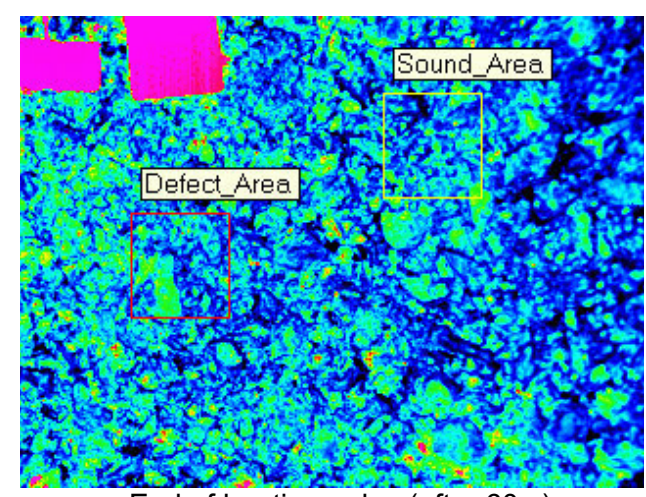

End of heating pulse (after $60 \mathrm{~s}$ )

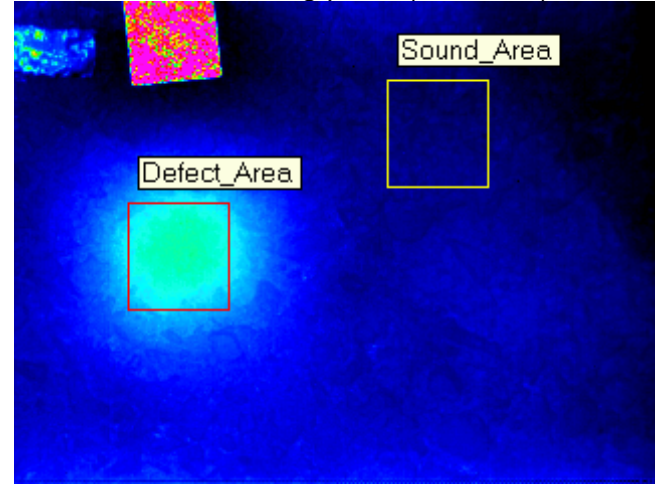

after $240 \mathrm{~s}$

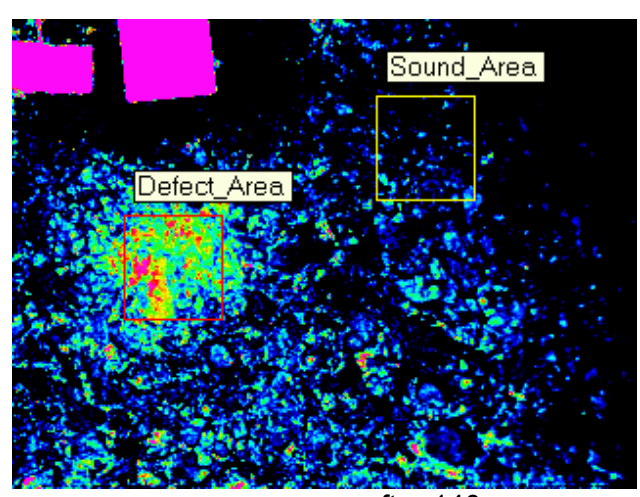

after $140 \mathrm{~s}$

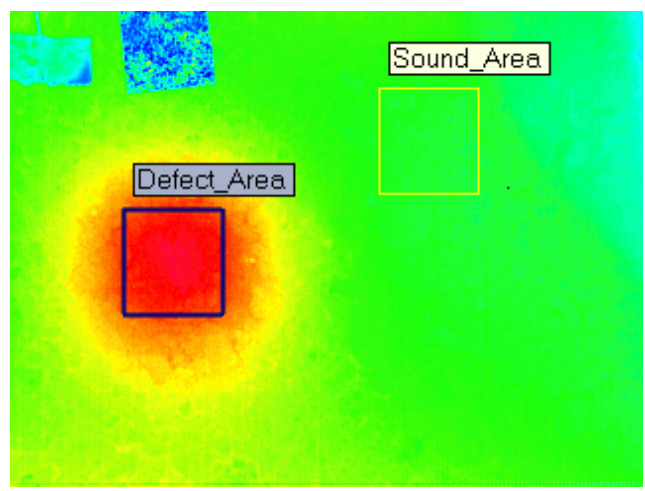

after $540 \mathrm{~s}$

Fig. 7. CEDIP. Jade I!l contrașt images - heating duration 1 minute ạt 2620 W. . $^{-2}$.- Square defeçt filled with aịr in the three defects pavement sample 


\subsubsection{Thermal conductivity numerical approach}

Temperature profiles in sound and defect region were analysed considering that the material behaves like a semiinfinite body of thermal effusivity $b$. Thus the variation of temperature $\theta(t)$ with time during a thermal excitation of power density $P$ can be expressed as proposed in ref. [5] in Eq. (2):

$$
\theta(t)=\alpha\left(1-\exp \left(\beta^{2} t\right) \operatorname{erfc}(\beta \sqrt{t})\right)
$$

With

$$
\left\{\begin{array}{l}
\alpha=\frac{P}{h} \\
\beta=\frac{h}{b}
\end{array}\right.
$$

In equation $3, h$ is the thermal exchange coefficient on the front side of the sample. This parameter is supposed to be constant during all the experiment. We must note here that the product of both parameters $\alpha$ and $\beta$ is independent of the heat exchange coefficient:

$$
\alpha \beta=\frac{P}{b}
$$

Parameters $\alpha$ and $\beta$ were identified by performing a least-square minimization using the Levenberg-Marquardt method. These estimations were carried out by considering experimental data in a time domain between 0 s (start of the excitation) and a time $t_{\mathrm{e}}$. Several estimations were performed for each value of $t_{\mathrm{e}}$ ranging from $60 \mathrm{~s}$ to $3600 \mathrm{~s}$ (end of the excitation) with a time increment of 60s. Then, the evolution of the product $\alpha \beta$ and of the estimation residual norm versus time $t_{\mathrm{e}}$ was analysed. If the material can be considered as a semi-infinite body, the product $\alpha \beta$ must remain constant and the residual norm must have a low value. If not, this is due to the presence of a region of different thermophysical properties inside the sample. The aim is here to define a characteristic time $t_{c}$, corresponding to the time when the thermal model becomes invalid. This method was alreadly proposed in a previous paper [6], where we showed that this characteristic time can be correlated to the position of the interface between the surface material and the second layer material (here the wood defect). The results are presented in figure 8.

In the case of the sound region, the product $\alpha \beta$ is nearly equal to $0.55 \mathrm{~K} . \mathrm{s}^{-1 / 2}$, excepted for lower $t_{\mathrm{e}}$ values, due to numerical instabilities. The power density $P$ during the considered experiment is equal to $770 \mathrm{~W} . \mathrm{m}^{-2}$. This value was obtained by calibration using a reference material (PVC plate) of known thermophysical properties and emissivity close to the asphalt one. This allows obtaining a thermal effusivity value of the asphalt material: $b=$ $1400 \mathrm{~W} \cdot \mathrm{s}^{1 / 2} \cdot \mathrm{m}^{-2} \cdot \mathrm{K}^{-1}$. This value is compatible with thermal effusivity values of some aggregates commonly used for the processing of road pavements (see [7]). Then, considering mean values of estimated parameters $\alpha$ and $\beta$, we can estimate the value of the heat exchange coefficient: $h=8.1-8.4 \mathrm{~W} \cdot \mathrm{m}^{-2} \cdot \mathrm{K}^{-1}$.

In the case of the defect region, the product $\alpha \beta$ remains constant for $t_{\mathrm{e}}$ values lower than $t_{\mathrm{c}}=900 \mathrm{~s}$. The value obtained for this parameter is close to the one obtained in the case of the sound region. For measurement times greater than $t_{\mathrm{c}}=900 \mathrm{~s}$, we observe a continuous increase of the product $\alpha \beta$ and of the residual norm. Considering on one hand a thermal diffusivity value $a$ of the asphalt material close to $4.5 \times 10^{-7} \mathrm{~m}^{2} . \mathrm{s}^{-1}$ (typical value for this kind of material), and on the other hand that the interface between the first and second layer influences the heat transfer for a Fourier number equal to 1 , then we can estimate the depth of the defect. In that case, the value obtained is equal to $2 \mathrm{~cm}$, i.e. greater than the theoretical value $(1.3 \mathrm{~cm})$.
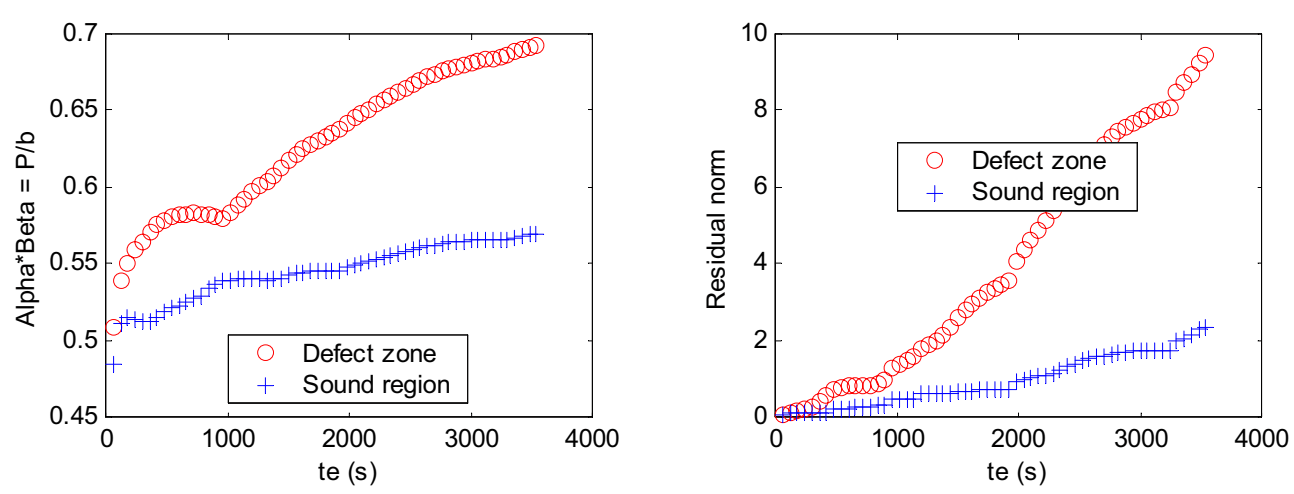

Fig. 8. Evolution of the estimated values of the produçt of parameters $\alpha$ and $\beta$ (left figure) and of the residual norm (right figure) for increasing measurement times $t_{e}$ 


\subsection{Numerical simulation results}

\subsubsection{Numerical model}

To complete experimental data, numerical simulations based on the finite volume method of heat transfers inside investigated samples were carried out using FLUENT TM. The modelling part thus consists in applying a step function of heating power of $400 \mathrm{~W}$ and duration equal to $600 \mathrm{~s}$ to the front face of the sample and in determining the temperature field on the surface and inside the sample as a function of time. The geometry of the sample considered is corresponding to the sample containing two defects. According to this geometry, we used a threedimensional non-structured meshing basis on tetrahedral cells realized under GAMBIT TM. The number of cells is 1892429. The mesh obtained is presented in figure 9 along with a view of the real pine wood defects included in the pavement sample. The thermal characteristics of materials, used for numerical simulations are presented in Table 3. Also, ambient temperature was considered as constant and equal to $20^{\circ} \mathrm{C}$, and a global heat exchange coefficient $h=10 \mathrm{~W} \cdot \mathrm{m}^{-2} \cdot \mathrm{K}^{-1}$ was considered for the front, rear and lateral faces of the sample.

Table 3: Thermophysical properties of materials used for numerical simulations

\begin{tabular}{|l|l|l|l|}
\hline Material & $\boldsymbol{k}\left(\mathbf{W} \cdot \mathbf{m}^{-1} \cdot \mathbf{K}^{-1}\right)$ & $\left.\rho \mathbf{k g} \cdot \mathbf{m}^{-\mathbf{3}}\right)$ & $\mathbf{C p}\left(\mathbf{J} \cdot \mathbf{k g}^{-1} \cdot \mathbf{K}^{-1}\right)$ \\
\hline Bitumen concrete & 1.41 & 2262 & 1255 \\
\hline Pine wood & 0.15 & 600 & 1900 \\
\hline
\end{tabular}
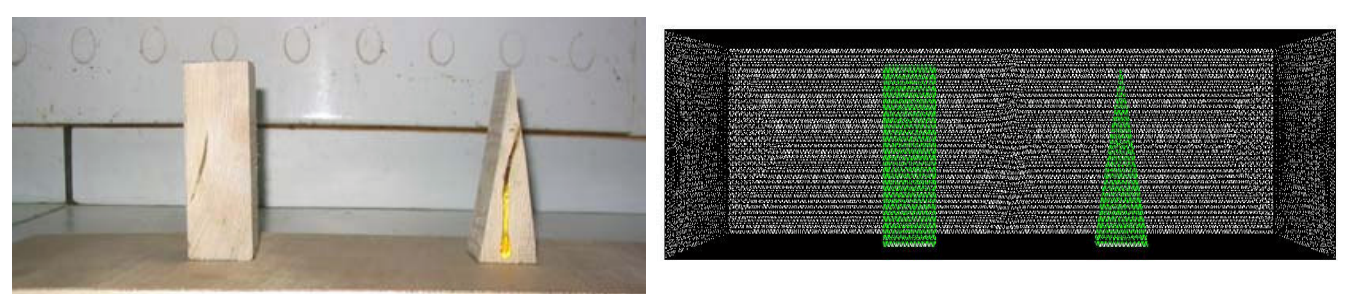

Fig. 9. View of defects considered for numerical simulations under FLUENT ${ }^{\mathrm{TM}}$ and the corresponding nonstructured mesh

\subsubsection{Results}

In figure 10, we present some results obtained by numerical simulation performed under FLUENT ${ }^{\mathrm{TM}}$, for an heating power of $400 \mathrm{~W}$ during $600 \mathrm{~s}$ : Different results obtained at different exposure times are reported. For each exposure time, we present the surface temperature, and the isotherm curves inside the sample. Before 300 s of heating, the defects are not appearing on the front face of the sample. We suppose that the heat flux does not still reach the defects for this exposure time. Beyond 300s of heating, we observe progressively the presence of a warm spot on the front face located above the parallelepiped defect. Heat propagation is modified in this region due to the wood thermal conductivity and induces an increase of local temperature. On the contrary, above the pyramidal defect, no significant change in the surface temperature is observed. This is due to the shape of this defect; in that case, heat flow lines are only slightly modified by the presence of this defect. This can be seen on isothermal curves representation: isothermal lines are not significantly modified by the presence of this defect, contrarily to the parallelepiped shape defect. These results confirmed experimental observations presented above.

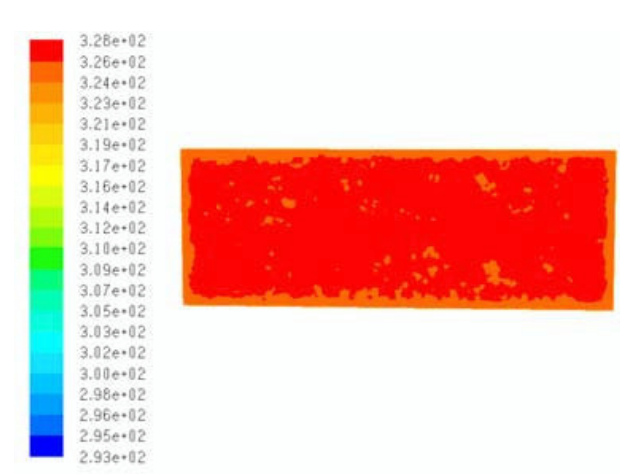

Front face surface temperature for $t=200$ s

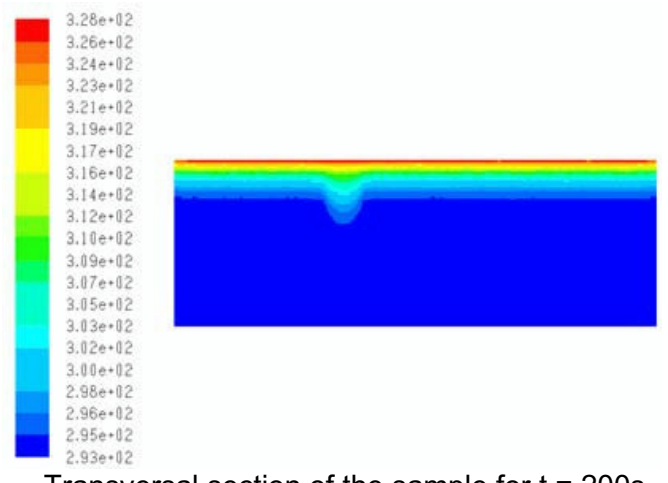

Transversal section of the sample for $t=200$ s 


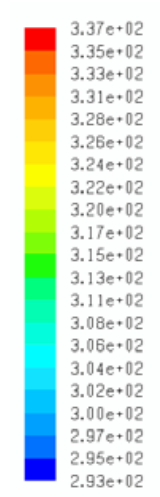

Front face surface temperature for $t=300$ s

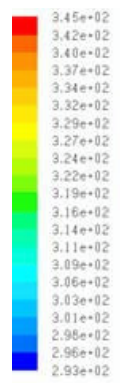

Front face surface temperature for $t=400$ s

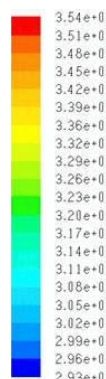

Front face surface temperature for $\mathrm{t}=600 \mathrm{~s}$

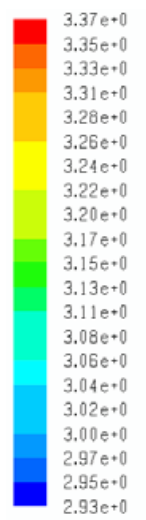

Transversal section of the sample for $t=300 \mathrm{~s}$

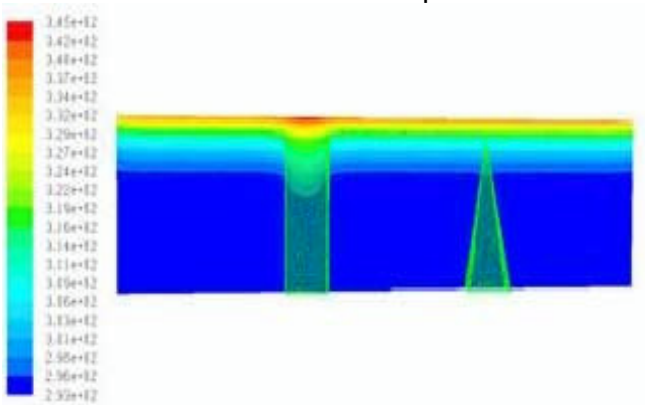

Transversal section of the sample for $t=400$ s
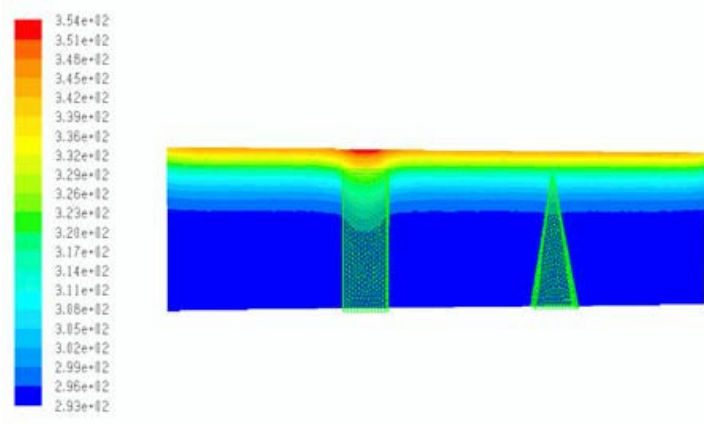

Transversal section of the sample for $\mathrm{t}=600 \mathrm{~s}$

Fig. 10. Froṇt face surface temperature (left) and isothermal curves (right) into the two defects sample section obtained by numerical simulation under FLUENT ${ }^{\mathrm{TM}}$. for different exposure times.t.

In figure 11 we present numerical and experimental temperature map obtained at 4 minutes after a 5 minutes heat pulse. On the numerical simulation the pyramid-shape pine wood defect is on the right of the image and on the left on the experimental one. On the numerical temperature map the difference of temperature between this defect and the sound part of the pavement sample is less than $0.2 \mathrm{~K}$. On the experimental one it is less than 0.4 K. But for experimental map defect shape and signature intensity are more noisy and affected by non uniform natural convection developed on the pavement sample surface but also by the quality of the NUC realized at the beginning of the trial with the CEDIP ${ }^{\circledR}$ Jade III IR camera. An another aspect that is not studied in this work is the local presence of more voids in the bitumen concrete sample around the defect due to mechanical behavior under compaction during pavement sample realization in laboratory. Such aspect may enhance the thermal signature of the defect in the infrared images acquired but it has still to be studied. So, at that stage of our study, without any information on the location of the pyramid-shape defect, its detection remains difficult and not established. 


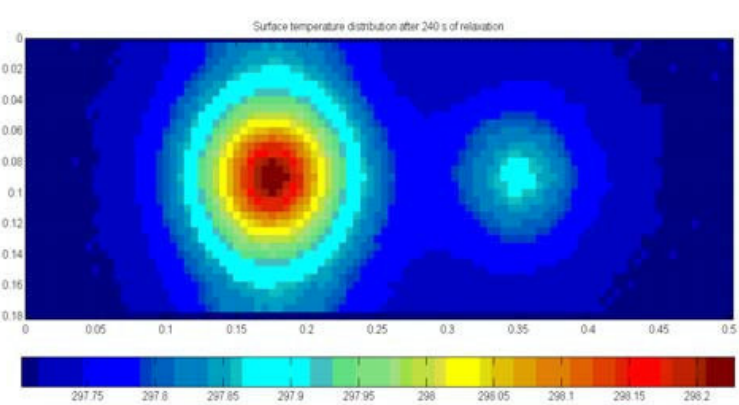

(a)Temperature map obtained by numerical simulation

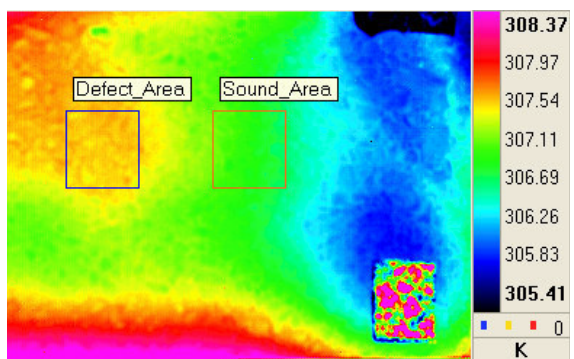

(b) Experimental temperature map

Fig. 11. Numerical and experimentạl temperature maps over the pyramid-shaped defect in pine wood ạt 4 minutes after the 5 minutes heat pulse

\section{Conclusion and perspectives}

As stated at the beginning of this study, the main objective was to evaluate to which extent active infrared thermography could be applied to detect road pavements defects. The step heating method was selected as a first one of investigation.

The step heating method was efficient to identify the presence of defects within this particular structure of road material. It has been shown that the heterogeneity of materials used in road pavements (porosity, variety of materials) could be bypassed. Defects down to $1.3 \mathrm{~cm}$ below the surface were detected. They appeared during the relaxation stage of the method. The analysis of the data using thermal contrast, either for the whole image or getting into details, indicated that all elements of the defect could be clearly extracted to properly identify it. On the other hand, defects with a narrow shape needed another analysis, and were not found. The step heating method was not the most adapted to this situation. Furthermore, the images from the FLIR ${ }^{\circledR} S 65$ camera were too noisy to run a proper investigation. Once these obstacles overtaken, some shape extraction and depths evaluation could be considered. Results obtained using the CEDIP ${ }^{\circledR}$ Jade III infrared lead to same conclusion. In that case, its spatial resolution coupled to its measurement performances has allowed us to discriminate the binder from the aggregates due to their different thermal behavior. On the other hand defect detection remains available during the heat relaxation phase for the pavement sample tested. Numerical simulations under FLUENT ${ }^{\mathrm{TM}}$ were helpful to complete our analysis and to understand limitation of such approach for pyramid-shape defects.

In the future we plan to test the PPT approach on our data but also to make some test on a real pavement structure built on the fatigue test track of the LCPC. On that one, different defects of different sizes and thickness have been implemented in the pavement structure during its construction and they will be submitted to heavy truck road traffic solicitation during several months.

\section{REFERENCES}

[1] X.P.V. Maldague. Theory and practice of infrared technology for non-destructive testing. John Wiley \& sons Inc., 2001.

[2] M. Larbi Youcef, A. Mazioud, L. Ibos, Y. Candau, P. Brémond, M. Piro, A. Filloux, A non destructive method for diagnostic of insulated building walls using infrared thermography, Thermosense XXIX 9-13 april 2007, Orlando.

[3] S. Datcu, L. Ibos, Y. Candau, S. Mattéï. Improvement of building wall surface temperature measurements by infrared thermography, Infrared Physics and Technology, Vol. 46, pp. 451-467, 2005.

[4] Ch. Maierhofer, R. Arndt, M. Röllig, C. Rieck, A. Walther, H. Scheel and B. Hillemeier, Application of impulse-thermography for non-destructive assessment of concrete structures, Cement \& Concrete Composite, vol 28, pp 393-401, 2006.

[5] V. Vavilov, Transient thermal NDT: conception in formulae, in Proceedings of QIRT 92, Paris 1992, pp. 229234, 1992.

[6] L.Ibos, M. Larbi Youcef, A. Mazioud, S. Datcu and Y. Candau, Non-destructive testing of building walls using active infrared thermography, in Proceedings of QIRT 06, Padova, 2006.

[7] French design manual for pavement structures, LCPC-SETRA publication, 1997. 
http://dx.doi.org/10.21611/qirt.2008.05_05_08 